\title{
INCLUSÃO, MEDIAÇÃO PEDAGÓGICA E ACESSIBILIDADE NO AMBIENTE EaD¹
}

\author{
Carolaine de Santana Garcia \\ Universidade Estadual Paulista - UNESP \\ carolainesgarcia@gmail.com \\ Aline Aparecida Alcântara da Silva \\ Universidade Estadual Paulista - UNESP \\ alinealcantaraapi@gmail.com \\ Elisa Tomoe Moriya Schlünzen \\ Universidade Estadual Paulista - UNESP \\ elisa.tomoe@unesp.br \\ Klaus Schlünzen Junior \\ Universidade Estadual Paulista - UNESP \\ klaus.junior@unesp.br
}

\section{RESUMO}

A construção de práticas educacionais inclusivas se configura como um dos principais desafios inerentes ao cenário educacional brasileiro, pois, pressupõe o desenvolvimento de práticas de ensino e aprendizagem para todos, a partir do respeito às especificidades e as diferenças de cada estudante. Assim, o paradigma educacional brasileiro tem passado por importantes reconfigurações, uma vez que a sociedade contemporânea tenta direcionar suas ações ao ideal de superação de desigualdades e desenvolvimento pleno do ser humano em todas as suas dimensões. Diante disso, a inclusão escolar se configura como um direito de todos os estudantes, em todos os níveis e modalidade de ensino, inclusive na Educação a Distância (EaD). Nessa perspectiva, é muito importante que os profissionais que desempenham o papel de mediadores pedagógicos, estejam atentos à importância em desenvolverem por meio de suas práticas, um trabalho que atenda às especificidades de todos os estudantes, acrescido a uma diversidade de recursos e condições que possibilitem, de fato, que todos possam aprender e participar equanimemente e com qualidade do processo educativo. Posto isto, com base na abordagem metodológica qualitativa, realizada por meio de uma pesquisa bibliográfica, o presente trabalho teve por objetivo refletir e analisar o papel do tutor no contexto da educação a distância, frente aos princípios de uma educação inclusiva.

\footnotetext{
${ }^{1}$ Este artigo é a versão expandida de uma comunicação oral apresentada no VII Simpósio de Educação Inclusiva e Adaptações/ V Simpósio Internacional de Educação a Distância. São Paulo, UNESP, 2019.
} 
Palavras-chave: Inclusão. Mediação Pedagógica. Acessibilidade. Educação a Distância. Ambientes Virtuais de Aprendizagem.

\title{
INCLUSION, PEDAGOGICAL MEDIATION AND ACCESSIBILITY IN THE EaD ENVIRONMENT
}

\begin{abstract}
The construction of inclusive educational practices is configured as one of the main challenges inherent to the Brazilian educational scenario, since it presupposes the development of teaching and learning practices for all, based on respect for the specificities and differences of each student. Thus, the Brazilian educational paradigm has undergone important reconfigurations, once contemporary society tries to direct its actions towards the ideal of overcoming inequalities and the full development of the human being in all its dimensions. Given this, school inclusion is configured as a right of all students, at all levels and teaching modality, including Distance Education (DE). In this perspective, it is very important that professionals who play the role of pedagogical mediators are aware of the importance of developing, through their practices, a work that meets the specificities of all students, plus a diversity of resources and conditions that enable in fact that everyone can learn and participate equally and with quality in the educational process. That said, based on the qualitative methodological approach, carried out through a bibliographic research, the present study aimed to reflect and analyze the role of the tutor in the context of distance education, in the face of the principles of inclusive education.
\end{abstract}

Keywords: Inclusion. Pedagogical Mediation. Accessibility. Distance Education. Virtual Learning Environments.

\section{INCLUSIÓN, MEDIACIÓN PEDAGÓGICA Y ACCESIBILIDAD EN EL ENTORNO EaD}

\section{RESUMEN}

La construcción de prácticas educativas inclusivas se configura como uno de los principales desafíos inherentes al escenario educativo brasileño, ya que presupone el desarrollo de prácticas de enseñanza y aprendizaje para todos, basadas en el respeto a las especificidades y diferencias de cada alumno. Así, el paradigma educativo brasileño ha sufrido importantes reconfiguraciones, ya que la sociedad contemporánea trata de dirigir sus acciones hacia el ideal de superar las desigualdades y el pleno desarrollo del 
ser humano en todas sus dimensiones. Dado esto, la inclusión escolar se configura como un derecho de todos los estudiantes, en todos los niveles y modalidades de enseñanza, incluida la educación a distancia (DE). En esta perspectiva, es muy importante que los profesionales que desempeñan el papel de mediadores pedagógicos, sean conscientes de la importancia de desarrollar a través de sus prácticas, un trabajo que cumpla con las especificidades de todos los estudiantes, además de una diversidad de recursos y condiciones que permitan de hecho, todos pueden aprender y participar por igual y con calidad en el proceso educativo. Dicho esto, basado en el enfoque metodológico cualitativo, llevado a cabo a través de una investigación bibliográfica, el presente estudio tuvo como objetivo reflejar y analizar el papel del tutor en el contexto de la educación a distancia, frente a los principios de la educación inclusiva.

Palabras claves: Inclusión. Mediación pedagógica. Accesibilidad. Educación a distancia. Entornos virtuales de aprendizaje.

\section{INTRODUÇÃO}

A inclusão escolar, de acordo com Mantoan (2004, p. 7) "é produto de uma educação plural, democrática e transgressora". Na ótica da autora, esta inclusão prevê uma inserção radical, completa e sistemática de todos os estudantes, sem qualquer exceção, no processo de aprendizagem.

Segundo Mantoan (2006, p. 9), "a perspectiva de se formar uma nova geração dentro de um projeto educacional inclusivo é fruto do exercício diário da cooperação e da fraternidade, do reconhecimento e do valor das diferenças". Tal concepção, não exclui ou menospreza a importância dos alunos aprenderem os conhecimentos historicamente produzidos pela humanidade, mas, reforça a necessidade de pensarmos em estratégias inovadoras de ensino que contemplem os diferentes ritmos e estilos de aprendizagem dos alunos, de modo que todos aprendam.

Consonante a isto, Sanches e Teodoro (2006, p. 74) afirmam que "para que a educação inclusiva seja uma realidade, para além de uma mudança de mentalidades, no que diz respeito ao acesso e ao sucesso da educação para todos, é necessário criar condições e recursos adequados a cada situação".

Diante dessa concepção de educação, que contemple as diferenças e as especificidades de todos os estudantes, sobretudo, dos Estudantes Público Alvo da Educação Especial (EPAEE), a saber, pessoas com deficiência, transtornos do espectro autista e altas habilidades/superdotação, surge à necessidade de criarmos ambientes de aprendizagem inclusivos, e pensarmos em recursos e estratégias que contemplem esses diferentes ritmos e estilos de aprendizagem, com vistas à superação de práticas discriminatórias, preconceituosas e excludentes. 
Posto isto, ao relacionarmos a educação inclusiva com a Educação a Distância, sustentada pelo paradigma educacional inclusivo, Rio (2018) nos destaca a importância de pensarmos e asseguramos uma diversidade de recursos e condições que possibilitem, de fato, que todos possam aprender e participar equanimemente, de modo, a superarmos a compreensão equivocada do ato de ensinar somente como mera transmissão dos conhecimentos historicamente acumulados e habilidades que precisam ser desenvolvidas, muito aquém disso, envolve a compreensão de que a formação integral do aluno requer também a disposição de um ambiente que favoreça o desenvolvimento de valores, da ética e da formação de atitudes, de maneira a promovermos um processo de ensino e aprendizagem a distância de qualidade e inclusivo.

\section{OBJETIVO}

O presente trabalho tem por objetivo refletir e analisar o papel do tutor no contexto da educação a distância e da importância deste profissional na construção de experiências, valores, princípios e compreensões teóricas e práticas que lhe permitam promover práticas pedagógicas que acolham as especificidades e necessidades educativas de todos os estudantes.

\section{FUNDAMENTAÇÃO}

A construção de práticas educacionais inclusivas se configura como um dos desafios inerentes ao cenário educacional brasileiro. Conforme Mantoan (2004, p. 39), "a inclusão é produto de uma educação plural, democrática e transgressora", assim, a Educação Inclusiva pressupõe o desenvolvimento de práticas de ensino e aprendizagem para todos, a partir do respeito às especificidades e às diferenças de cada um.

A Política Nacional de Educação Especial na Perspectiva da Educação Inclusiva (BRASIL, 2008), prevê e ressalta os direitos de acesso, participação e permanência dos EPAEE em todos os níveis e modalidades de ensino. Todavia, tais ações, orientam sobre a importância do planejamento e da organização de recursos e serviços que atendam às necessidades educacionais dos alunos, a fim de que, de fato, tenhamos uma escola de qualidade para todos (BRASIL, 2008).

À vista disso, a Resolução $n^{\circ}$ 2/2001 instituiu as Diretrizes Nacionais para a Educação Especial na Educação Básica (BRASIL, 2001), em que determinam que os sistemas de ensino tenham por obrigatoriedade matricular todos os alunos, sobretudo os alunos com deficiência. Em vista disso, ampliou-se o caráter de Educação Especial para a realização do AEE, de modo a complementar ou suplementar à formação dos EPAEE nas classes comuns. 
A Resolução n 4/2009 sobre o AEE, em seu artigo segundo, esclarece que,

Art. $2^{\circ} \mathrm{O}$ AEE tem como função complementar ou suplementar a formação do aluno por meio da disponibilização de serviços, recursos de acessibilidade e estratégias que eliminem as barreiras para sua plena participação na sociedade e desenvolvimento de sua aprendizagem. (BRASIL, 2009, p. 11).

Ainda, segundo a Resolução $n^{\circ} 4 / 2009$, este serviço é destinado aos:

I - Alunos com deficiência: aqueles que têm impedimentos de longo prazo de natureza física, intelectual, mental ou sensorial.

II - Alunos com transtornos globais do desenvolvimento: aqueles que apresentam um quadro de alterações no desenvolvimento neuropsicomotor, comprometimento nas relações sociais, na comunicação ou estereotipias motoras. Incluem-se nessa definição alunos com autismo clássico, síndrome de Asperger, síndrome de Rett, transtorno desintegrativo da infância (psicoses) e transtornos invasivos sem outra especificação.

III - Alunos com altas habilidades/superdotação: aqueles que apresentam um potencial elevado e grande envolvimento com as áreas do conhecimento humano, isoladas ou combinadas: intelectual, liderança, psicomotora, artes e criatividade. (BRASIL, 2009, p. 1)

Nesse sentido, o AEE deve ser realizado, prioritariamente, na Sala de Recursos Multifuncionais (SRM), na própria escola do atendido, ou em outra escola próxima, e também em centros especializados que ofereçam este serviço. Sendo assim, o AEE se configura como um serviço de fundamental importância no processo de desenvolvimento e aprendizagem dos EPAEE, perpassando todos os níveis e etapas, bem como modalidades de ensino, portanto, compreendendo da educação básica ao ensino superior.

Sendo assim, embora a legislação assegure a inclusão dos estudantes com deficiência nas classes comuns para o ensino regular com o apoio do serviço de atendimento educacional especializado o maior desafio está na mudança de atitudes e mentalidade em relação à inclusão. Mantoan (2004, p. 40) alerta que "[...] os argumentos pelos quais a escola tradicional resiste à inclusão [...] refletem a sua incapacidade de atuar diante da complexidade, da diversidade, da variedade, do que é real nos seres e nos grupos humanos".

No dizer de Carvalho (2004, p. 77),

A Letra das leis, os textos teóricos e os discursos que proferimos asseguram os direitos, mas o que os garante são as efetivas ações, na medida em que se concretizam os dispositivos legais e todas as deliberações contidas nos textos de políticas públicas. Para tanto, mais que prever há que prover recursos de toda a ordem, permitindo que os direitos humanos sejam respeitados, de fato. Inúmeras são as providências políticas, administrativas e financeiras a serem tomadas, para que as escolas, sem discriminações de qualquer natureza, acolham a todas as crianças, independentemente de suas condições físicas, intelectuais, sociais, emocionais, linguísticas ou outras. 
Ademais, Mantoan (2006, p. 12) ressalta que, "um novo paradigma do conhecimento está surgindo das interfaces e das novas conexões que se formam entre saberes outrora isolados e partidos e dos encontros da subjetividade humana com o cotidiano, o social, o cultural".

De acordo com Schlünzen (2015, p. 80), o pressuposto da Educação Inclusiva independente no nível ou modalidade em que ocorra, envolve mudanças significativas:

[...] especialmente quando, no caso das pessoas com deficiências, esse uso possibilita mobilidade, autonomia e acessibilidade, de maneira que as diferenças físicas, sensoriais e/ou motoras não sejam evidenciadas, faz com que as estratégias deformação em nível inicial ou continuado sejam repensadas, ou melhor, ressignificadas.

No Brasil, o ensino na modalidade EaD, teve seu início por volta do século XX, a partir das atividades do Instituto Universal Brasileiro, em 1941. Sendo um marco histórico a criação da Secretaria de Educação a Distância (SEED) do Ministério da Educação (MEC) em 1996, deste modo, foram desenvolvidas diversas ações e projetos, proporcionando assim o uso mais intensivo das Tecnologias, sendo elas voltadas para a formação de professores como também a sua introdução como modalidade de Ensino.

Para Nova e Alves (2003), a EaD é vista como uma modalidade de ensino, onde os estudantes não estão atrelados a uma sala de aula, havendo a criação de sistemas de ensino por meio de veículos de comunicação diversos (correio, rádio e televisão), atendendo uma parcela da população que não têm a oportunidade de frequentar esses espaços físicos regularmente.

Deste modo, compreendemos que a EaD pode sim proporcionar e ser um ambiente inclusivo, por meio da capacitação e disseminação de informações.

A Educação a Distância ganhou nos últimos anos o caráter democrático, ao considerar as características geográficas, econômicas e sociais de nosso país, difundindo o conhecimento apesar da distância, oportunizando assim, as pessoas das diferentes regiões brasileiras o acesso de forma quase igualitária. (SILVA, 2017, p.172).

Logo, dentro deste contexto educativo, a EaD pode contribuir para a Inclusão desde que esteja permeada pela Acessibilidade, para que isso ocorra, devemos pensar desde o ambiente/plataforma em que se desenvolvem os cursos, até mesmo no processo de mediação do professor/tutor, à vista disso, Cechinel (2000, p. 14), nos diz que o professor tutor-mediador possui a função de ser um "facilitador, mediador, motivador, orientador e avaliador da aprendizagem". 


\section{METODOLOGIA}

O presente estudo buscou refletir e analisar o papel do professor-tutor no contexto da educação a distância sob a perspectiva inclusiva, para tanto, nos pautamos em uma abordagem metodológica qualitativa de pesquisa, pois nos permite refletir sobre os múltiplos aspectos que envolvem o fenômeno estudado.

Richardson (1999, p.80) evidência que os dados na pesquisa qualitativa "[...] podem descrever a complexidade de determinado problema, analisar a interação de certas variáveis, compreender e classificar processos dinâmicos vividos por grupos e possibilitar, em maior profundidade, o entendimento das particularidades do comportamento dos indivíduos".

Dessa forma, segundo Strauss (2008, p. 23) quando nos referimos à pesquisa qualitativa podemos caracterizá-la como:

Qualquer tipo de pesquisa que produza resultados não alcançados através de procedimentos estatísticos ou de outros meios de quantificação. Pode se referir à pesquisa sobre a vida das pessoas, experiências vividas, comportamentos, emoções e sentimentos, e também à pesquisa sobre funcionamento organizacional, movimentos sociais, fenômenos culturais e interação entre nações.

Consonante a isto, Sandín (2010, p.127) destaca que essa abordagem de pesquisa pode ser vista como uma:

\footnotetext{
Atividade sistemática orientadaà compreensão em profundidade de fenômenos educativos e sociais, à transformação de práticas e cenários socioeducativos, à tomada de decisões e também descobrimento e desenvolvimento de um corpo organizado de conhecimentos.
}

Sendo assim, os resultados obtidos no decorrer deste estudo, não são mensuráveis, pois tem em seu pretenso desvelar questões repletas de significados, usos, valores e atitudes muito particulares aos sujeitos envolvidos, o que também requer o estabelecimento de uma relação indissociável entre pesquisador e pesquisado (MINAYO, 1994).

Para tanto, associado à abordagem qualitativa, este estudo se caracteriza como uma pesquisa bibliográfica. Sobre a pesquisa bibliográfica, Gil (2002, p.44), nos elucida que, "[...] a pesquisa bibliográfica é desenvolvida com base em material já elaborado, constituído principalmente de livros e artigos científicos".

Dessa forma, o material bibliográfico utilizado relacionado à Educação Inclusiva e Acessibilidade, foram encontrados em livros e artigos científicos de diferentes autores como Carvalho (2004), Mantoan (2004; 2006), Sanches e Teodoro (2006) e Schlünzen (2015). Desse modo, o aporte teórico relacionado à Educação a Distancia e a Mediação Pedagógica, teve como base os estudos 
de autores como Cechinel (2000), Alves (2003), Nova;Silva (2017), Rio (2018) e Schlünzen Junior (2018).

Assim, foi possível conhecermos e levantarmos as contribuições científicas relacionadas a esses temas, assim como dados que trazem definições coerentes com a prática, e que, portanto, nos possibilitam tecer reflexões a partir deste estudo.

\section{RESULTADOS E DISCUSSÃO}

No cenário educacional brasileiro, a Educação a Distância passou a assumir nos últimos anos um caráter cada vez mais democrático e transgressor, ao buscar oportunizar a todas as pessoas o acesso ao conhecimento.

Dado o exposto, compreendemos que para que esse processo de ensino e a aprendizagem consiga de fato, viabilizar oportunidades educacionais a todos os estudantes, considerando as especificidades dos seus estudantes, seus interesses, condições de vida e de trabalho e necessidades, o professor, na condição de mediador, desempenha um papel fundamental, não sendo possível, portanto, separar os aspectos técnicos e pedagógicos da sua prática.

À vista disto, algumas medidas podem ser adotadas, no intuito de desenvolvermos uma mediação pedagógica efetiva e de qualidade, e, sobretudo, em uma perspectiva inclusiva. Antes o exposto, Rio (2018) nos esclarece que uma das primeiras medidas a serem adotadas, diz respeito a conhecermos os estudantes, suas especificidades e necessidades educativas, a fim de realizarmos um mapeamento das dificuldades apresentadas para a prática da mediação, com vistas a auxiliarmos nossos alunos a superá-las.

Assim, uma estratégia muito favorável a esse processo, seria o provimento de um espaço onde todos os estudantes pudessem se apresentar e descrever seu perfil, de modo que, a partir das informações coletadas, houvesse um mapeamento das características especificas de cada cursista, visto que deste modo podemos identificar similaridades e as diferenças, favorecendo assim uma aproximação entre os próprios estudantes, como também do tutor com a turma de estudantes.

Ademais, outro aspecto a ser considerado, diz respeito à comunicação e a linguagem, pois no que tange ao ensino $\mathrm{EaD}$, é primordial que a comunicação entre tutor e cursista ocorra de maneira ativa, fator este, que ganha destaque ao relacionarmos aos aspectos da acessibilidade pedagógica e comunicacional.

Desse modo, deve ser evitado o envio de mensagens ambíguas e com excesso de informações, valorizando uma linguagem acessível, que objetive a clareza e objetividade, de maneira a ser facilmente compreendida pelos estudantes, além disso, o mediador pedagógico deve se colocar sempre à disposição para esclarecer e solucionar possíveis dúvidas ou diante da necessidade de mais alguma informação. 
Dessa forma, o acompanhamento do estudante visando um assessoramento contínuo se comprova como uma prática eficiente e inclusiva, de modo que, assim, possamos verificar suas dificuldades, habilidades e potencialidades, possibilitando que o estudante se sinta amparado, que mesmo a distância, pode contar com um suporte para auxiliá-lo.

Por fim, mas não menos importante, a fase de Avaliação e Feedback se constitui como uma etapa muito importante, na qual devemos buscar por subsídios palpáveis que expressem o processo de construção do conhecimento dos alunos. Para tanto, deve-se ter em conta a necessidade de uma avaliação que ocorre de modo processual, por meio do acompanhamento do cumprimento dos objetivos propostos para cada atividade, acrescido a prática de oferecer feedbacks que expressem os conhecimentos aprendidos e indicando o que ainda precisa ser desenvolvido e aprimorado.

Acrescido às práticas que podem ser adotadas pelos tutores na interação com os cursistas encontram-se presentes a acessibilidades do material, recursos e plataformas que se utiliza na EaD, pois, para se obter êxito no processo inclusivo é necessário que caminhem juntos. Pois, as dificuldades encontradas por essas pessoas na aprendizagem são provocadas pela inadequação dos Ambientes Virtuais de Aprendizagem (AVA), dos materiais didáticos e das metodologias de ensino, causando barreiras na sua participação no processo educacional, privando-as da igualdade de oportunidades de aprender em condição de similaridade com as demais pessoas (SCHLÜNZEN JUNIOR, 2018).

Nesta conjuntura, a acessibilidade tem o papel de derrubar as barreiras para que os estudantes possam participar e ter autonomia com relação ao seu próprio processo de aprendizagem. Portanto, não se deve esperar que os EPAEE se adequem as estruturas já existentes, a Legislação Brasileira, já infere que os sistemas de ensino devam atender a todos sem distinção e se adequar e se estruturar para dar conta da demanda e particularidade dos alunos. Logo, é imprescindível que os materiais e recursos sejam adaptados em Libras, com audiodescrição, a utilização uso de softwares e plataformas com estes e outros recursos de acessibilidade.

Portanto, o uso de recursos de acessibilidade, aliada a uma prática ativa de mediação, podem ser elementos decisivos na construção de um ambiente inclusivo, que propiciem a aprendizagem e desenvolvimento profissional inclusivo e com qualidade para todos.

\section{CONSIDERAÇÕES FINAIS}

Tomando por base a importância de reconfigurações no paradigma educacional e, objetivando a superação de desigualdades e a participação de todos os 
estudantes no processo educativo, o presente trabalho buscou ressaltar a importância de pensarmos e criamos condições que promovam a aprendizagem e possibilitem aos educandos o desenvolvimento de suas potencialidades.

Nessa perspectiva, é muito importante que os profissionais que desempenham o papel de mediadores pedagógicos estejam atentos a necessidade de desenvolverem um trabalho, que atenda às especificidades de todos os estudantes e que oferece uma educação de qualidade para todos.

Ainclusão efetiva vai além da concepção de conceitos e do uso de recursos, que são de grande importância neste contexto, porém, a atuação do professor-tutor deve superar essa dualidade, sendo peça chave para favorecer a participação de todos os alunos, onde deve se considerar a potencialidades dos estudantes, como a sua subjetividade, visto que o processo de ensino e aprendizagem pode se dar pelas trocas de experiências, promovendo o "estar junto virtual", onde a distância os separa, mas o destino será o mesmo.

Desta forma, temos que um bom tutor, capacitado e ciente das potencialidades dos seus estudantes com ou sem deficiências, será um bom mediador entre os conhecimentos que se pretende construir e o aluno, de modo a construir práticas educacionais que valorizem as especificidades dos estudantes, considerando suas habilidades e potencialidades, auxiliando e promovendo o desenvolvimento de sua autonomia e aprendizagem significativa.

Portanto, ao atingirmos nosso objetivo de refletir e analisar o papel do tutor no contexto da educação a distância, chegamos conclusão que mais do que acessibilidade nos ambientes EaD, a participação do professor-tutor se torna fundamental para o processo inclusivo, sendo ele a ponte que pode interligar o real e o virtual, onde dispõe seus conhecimentos pedagógicos, tecnológicos e humano a serviço do ensino e da aprendizagem.

\section{REFERÊNCIAS}

BRASIL. Ministério da Educação. Diretrizes Nacionais para a Educação Especial na Educação Básica. MEC; SEEP; 2001.

BRASIL. Ministério da Educação. Política Nacional de Educação Especial na perspectiva da Educação Inclusiva. MEC; SEEP; 2008.

BRASIL. Conselho Nacional de Educação. Câmara de Educação Básica. Resolução CNE/ CEB n. ${ }^{\circ}$, de 2 de outubro de 2009. Institui Diretrizes Operacionais para o Atendimento Educacional Especializado na Educação Básica, modalidade Educação Especial. Diário Oficial da União, 5 de outubro de 2009. 
CARVALHO, R. É. Educação Inclusiva: Com os Pingos nos "is". Porto Alegre: Mediação, 2004.

CECHINEL, J. C. Manual do Tutor. Florianópolis: Udesc, 2000.

GIL, A. C. Como elaborar projetos de pesquisa. São Paulo: Atlas, 2002.

MANTOAN, M. T. E. Inclusão escolar: O que é? Por quê? Como fazer? São Paulo: Editora Moderna, 2006.

MANTOAN, M. T. E. O direito de ser, sendo diferente, na escola. Revista CEJ, v. 8, n. 26, jul./set. 2004.

MANTOAN, M. T. E. Caminhos Pedagógicos da Educação Inclusiva. In: GAIO, R.; MENEGHETTI, R.G.K. (org.). Caminhos Pedagógicos da Educação Especial. Petrópolis: Editora Vozes, 2004.

MINAYO, M. C. S. Ciência, técnica e arte: o desafio da pesquisa social. In: MINAYO, M. C. S.(org.). Pesquisa social: teoria, método e criatividade. 18. ed. Petrópolis: Vozes, 1994.

NOVA, C. ALVES, L. Educação a distância: Limites e Possibilidades. Texto publicado no livro Educação a distância: uma nova concepção de aprendizado e interatividade. São Paulo: Futura, 2003.

RICHARDSON, R. J. Pesquisa social: métodos e técnicas. 3. ed. São Paulo: Atlas, 1999.

RIOS, G. A. Inclusão pedagógica: conceituação a partir de uma experiência na educação superior a distância. 2018. 200 f. Tese (Doutorado em Educação) Universidade Estadual Paulista “Júlio de Mesquita Filho", Presidente Prudente, 2018.

SANDÍN, Maria Paz Esteban. Pesquisa qualitativa em educação: fundamentos e tradições. Porto Alegre: AMGH, 2010.

SANCHES, I; TEODORO, A. Da integração à inclusão escolar: cruzando perspectivas e conceitos. Revista Lusófona de Educação, 63-83 2006.

SILVA, M.M. O processo de inclusão nos cursos de ead. Disponível em: https:// periodicos.ufersa.edu.br/index.php/includere/article/view/7406. Acesso em: 25 ago. 2019.

SCHLÜNZEN, E. T. M. Abordagem Construcionista, Contextualizada e Significativa: formação, extensão e pesquisa em uma perspectiva inclusiva. 2015. 200f. Tese (Livre Docência). Universidade Estadual Paulista, Faculdade de Ciências e Tecnologia, Presidente Prudente/São Paulo. 
SCHLÜNZEN JUNIOR, K.; SCHLUNZEN, E. T. M.; D. A. N.; MALHEIRO, C. A. L.; SANTOS. Acessibilidade e Inclusão em Contexto on Line. Brasil: Capes, NEaD - Unesp; Portugal: UAb, 2018. (Material do curso Formação de Formadores para a mediação on-line).

STRAUSS A, C. J. Pesquisa qualitativa: técnicas e procedimentos para o desenvolvimento de teoria fundamentada. 2. ed. Porto Alegre (RS): Artmed; 2008.

\section{BIOGRAFIA DOS AUTORES}

CAROLAINE DE SANTANA GARCIA - Graduada em Licenciatura em Pedagogia com habilitação em Gestão Escolar da Educação Básica, pela Universidade Estadual Paulista Júlio de Mesquita Filho (UNESP), campus de Presidente Prudente, SP. Atualmente é estudante de Mestrado em Educação pelo Programa de Pós-Graduação em Educação (PPGE) da FCT/UNESP, com bolsa concedida pela Coordenação de Aperfeiçoamento de Pessoal de Nível Superior (CAPES), do Ministério da Educação (MEC). Atua como colaboradora do Centro de Promoção para Inclusão Digital, Escolar e Social (CPIDES), localizado na Faculdade de Ciências e Tecnologia-UNESP.

ALINE APARECIDA ALCÂNTARA DA SILVA - Graduada em Licenciatura em Matemática pelo Centro Universitário de Maringá (UniCesumar). Atualmente mestranda em Educação pelo Programa de Pós-Graduação em Educação (PPGE), com bolsa concedida pela Coordenação de Aperfeiçoamento de Pessoal de Nível Superior (CAPES), do Ministério da Educação (MEC) e graduanda em Licenciatura em Pedagogia da Universidade Estadual Paulista Júlio de Mesquita Filho (FCT/UNESP) campus de Presidente Prudente, SP. Atua como colaboradora do Centro de Promoção para Inclusão Digital, Escolar e Social (CPIDES).

ELISA TOMOE MORIYA SCHLÜNZEN - Possui graduação em Licenciatura Plena em Matemática pela Universidade Estadual Paulista - UNESP (1985), mestrado em Engenharia Elétrica pela Universidade Estadual de Campinas (1994) e doutorado em Educação (Currículo) pela Pontifícia Universidade Católica de São Paulo (2000). É Livre-Docente em "Formação de Professores para uma Escola Digital e Inclusiva" pela UNESP (2015). Atualmente é professora adjunta voluntária na UNESP e professora do Programa de Pós-Graduação da Universidade do Oeste Paulista (Unoeste). De 2010 a 2018 foi coordenadora do Centro de Promoção para a Inclusão Digital, Escolar e Social (CPIDES). 
KLAUS SCHLÜNZEN JUNIOR - Licenciado em Matemática pela Universidade Federal de Santa Catarina, mestre em Ciência da Computação pela Universidade Estadual de Campinas e doutor em Engenharia Elétrica pela Universidade Estadual de Campinas. Professor livre-docente em Informática e Educação pela Universidade Estadual Paulista - UNESP, com estágio de Pós-doutoramento na Universitat de Barcelona e becário da Fundación Carolina. É professor efetivo do Departamento de Estatística, do programa de Pós-graduação em Educação da FCT/Unesp, coordenador geral do Programa de Mestrado Profissional em rede em Educação Inclusiva (PROFEI) e coordenador do Centro de Promoção para a Inclusão Digital, Escolar e Social (CPIDES).

Data de recebimento: 01/09/2020

Data de aprovação: 23/10/2020 